\title{
Titanium(IV)-induced cristobalite formation in titanosilicates and its potential impact on catalysis
}

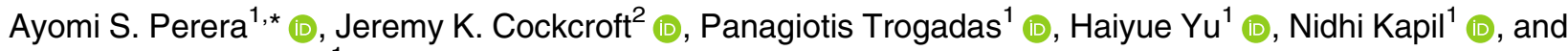 \\ Marc-Olivier Coppens ${ }^{1, \star}$ (i) \\ ${ }^{1}$ Department of Chemical Engineering, Centre for Nature Inspired Engineering, University College London, Torrington Place, \\ London WCIE 7JE, UK \\ ${ }^{2}$ Department of Chemistry, University College London, 20 Gordon Street, London WC1H OAJ, UK
}

Received: 2 July 2018

Accepted: 27 August 2018

Published online:

6 September 2018

(C) The Author(s) 2018

\begin{abstract}
Cristobalite, a crystalline form of silica, is shown to be formed within an amorphous titanosilicate, at previously unknown conditions. Mesoporous titanosilicate microspheres (MTSM) were synthesized as efficient catalysts for the epoxidation of cyclohexene with tert-butyl hydroperoxide. High-resolution transmission electron microscopy revealed the presence of crystals in this predominantly amorphous material, after calcination at $750{ }^{\circ} \mathrm{C}$. When calcined at $800{ }^{\circ} \mathrm{C}$, the crystals were identified via PXRD as predominantly cristobalite, which possibly marks its first observation in titanosilicates at such a low temperature, without adding any alkali metals during synthesis. Catalytic experiments conducted with MTSM materials calcined at temperatures varying from 650 to $950{ }^{\circ} \mathrm{C}$, reveal that the amount of cristobalite formed increases with temperature, and that it has a significant impact on the pore structure, and, remarkably, correlates with the catalytic activity of titanosilicates.
\end{abstract}

\section{Abbreviations}

TBHP Tert-butyl hydroperoxide

TEOS Tetraethyl orthosilicate

DM Doehlert matrix

PXRD Powder X-ray diffraction

HRTEM High resolution transmission electron microscopy

NLDFT Non-local density functional theory

BET Brunauer-Emmett-Teller

BJH Barrett-Joyner-Halenda
EDX Energy dispersive X-ray spectroscopy

XPS X-ray photoelectron spectroscopy

\section{Introduction}

Titanosilicates are versatile materials with applications in catalysis, ion-exchange and membrane technology in petrochemical, pharmaceutical, food and

\footnotetext{
Address correspondence to E-mail: ayomi.perera@ucl.ac.uk; m.coppens@ucl.ac.uk
} 
water purification industries [1, 2]. In particular, they are commercially valuable as highly selective heterogeneous catalysts for oxidation of alkenes, to form industrially relevant organic epoxides and alcohols, which are precursors for various agrochemicals, food preservatives and pesticides [3-5]. Titanosilicates hold significant advantages over other conventional catalysts in these processes, as, in addition to product selectivity, they can also be used under mild reaction conditions, to form environmentally benign byproducts, are hydrothermally stable, and generate high product yields and substrate conversions [6-9]. Their synthesis can also be tailored to form micro-, meso- or hierarchical micromesoporous and layered structures, for the catalysis of a wide range of organic substrates [2, 6, 10-12].

The customization of the physical structure of titanosilicates can be achieved via temperature-dependent phase transformations, in order to generate various frameworks, and layered structures, that target specific catalytic reactions or ion-exchange applications [13-16]. Calcination is often used in such processes, to burn off templating agents and other impurities [17]. In addition, with increasing calcination temperature, titanosilicates transform between various structural phases, such as amorphous-tocrystalline-to-layered [14] or crystalline-to-amorphous-to-layered [13]. Understanding the chemical and physical conditions of such transformations is a key factor in the effective synthesis of titanosilicates.

Phase transitions are usually observed when ionic forms of alkali metals, such as $\mathrm{Na}$ and $\mathrm{K}$, are incorporated during the synthesis of titanosilicates [13-16, 18]. In silica materials, it is well known that the presence of Group 1 elements (such as $\mathrm{Na}$ and $\mathrm{Li}$ ) facilitate phase separations by significantly lowering the transition temperature [19] and similar effects are seen in titanosilicates. In addition, the presence of crystalline anatase $\mathrm{TiO}_{2}$ phases commonly contributes to the phase heterogeneity of titanosilicates $[17,20]$. Increasing the Ti content during synthesis of titanosilicates has been shown to increase the formation of anatase, a crystalline form of $\mathrm{TiO}_{2}$ [21]. It has long been established that the catalytic activity of titanosilicates is brought about by the isolated tetrahedral $\mathrm{Ti}^{4+}$ sites within the silica matrix; [22-24] thus, the presence of catalytically inactive $\mathrm{TiO}_{2}$ phases is highly undesirable. Furthermore, $\mathrm{TiO}_{2}$ causes the decomposition of oxidants used in titanosilicate-catalyzed reactions [25], adding to further catalytic inefficiency. Therefore, minimizing the formation of crystalline $\mathrm{TiO}_{2}$ phases is crucial in developing effective titanosilicate catalysts. What is less understood, however, is whether the presence of crystalline silica phases, such as cristobalite, has any effect on the catalytic activity of titanosilicates. This is partly due to the fact that such phases are present in small amounts and are not often identified.

Cristobalite is a crystalline form of silica, which is naturally found in volcanic rocks [26-28]. Pure silica gels can form cristobalite by phase transformation at temperatures as high as $\sim 1470-1705^{\circ} \mathrm{C}$ [29-31]. However, with the presence of alkali metals such as $\mathrm{Na}$ and Li (usually found in volcanic rocks), this temperature can be reduced to as low as $900{ }^{\circ} \mathrm{C}$ [19, 32]. Reports of cristobalite formation in titanosilicates are rare, and when reported, cristobalite was present in small quantities and was found only when sources of $\mathrm{Na}$ were included in stoichiometric amounts during the synthesis of titanosilicates [33-35]. There is no evidence in the literature indicating that the presence of $\mathrm{Ti}^{4+}$ itself has any effect on the formation of such crystalline silica phases.

The goals of this study were: (1) to investigate the factors that induce the formation of cristobalite formed in titanosilicates at low temperatures, without the incorporation of other phase transition inducing agents and (2) whether these cristobalite phases have an effect on the catalytic activity of titanosilicates. The results obtained provide evidence of formation of cristobalite at temperatures much lower than those reported previously, without the addition of alkali metals or organic ligands. The impact of the crystalline phases on the catalytic activity of titanosilicates was thoroughly investigated and discussed. These findings may be of critical importance in understanding factors that affect catalytic activity of titanosilicates, as well as provide insight into the importance of controlling calcination conditions in large-scale synthesis.

\section{Materials and methods}

\section{Synthesis of titanosilicate microspheres}

Synthesis was based on previously published procedures [17, 36]. All chemicals were purchased from Sigma-Aldrich and used without further purification. 


\section{General procedure}

$1.0 \mathrm{ml}$ of Ti(IV) n-butoxide (99\%) was added dropwise to $30.0 \mathrm{ml}$ of DI water $(18.2 \mathrm{M} \Omega)$, at $4{ }^{\circ} \mathrm{C}$, under magnetic stirring, in order to form $\mathrm{Ti}(\mathrm{OH})_{4}$ precipitate. The precipitate was filtered under vacuum and washed with $\mathrm{DI}$ water. $\mathrm{Ti}(\mathrm{OH})_{4}$ was then dissolved in $4.0 \mathrm{ml}$ of $4 \mathrm{~N} \mathrm{HNO}_{3}$ to produce the active species $\mathrm{TiO}\left(\mathrm{NO}_{3}\right)_{2}$. $\mathrm{TiO}\left(\mathrm{NO}_{3}\right)_{2}$ was then mixed with a solution of $6.6 \mathrm{ml}$ tetraethyl orthosilicate (TEOS, 98\%) and $2.0 \mathrm{ml}$ of ethanol and stirred vigorously for $30 \mathrm{~min}$ to form isolated $\mathrm{Ti}^{4+}$ sites in the silica matrix. Next, surfactant templating was carried out to create mesoporous microspheres: The above solution was transferred to a mixture of $26.1 \mathrm{~g}$ kerosene and $7.9 \mathrm{~g}$ Span 80, and homogenized with an Ultra-Turrax homogenizer, at $3000 \mathrm{rpm}$ for $2 \mathrm{~h}$, at $80^{\circ} \mathrm{C}$. The microspheres formed were then vacuum-filtered and washed with acetone and DI water, followed by drying at $80{ }^{\circ} \mathrm{C}$ for $2 \mathrm{~h}$. This synthesis procedure was repeated seven times. Finally, the seven samples thus obtained were calcined at different temperatures, namely $650,700,750,800,850,900$ and $950{ }^{\circ} \mathrm{C}$ for $6 \mathrm{~h}$. Hereafter, these samples will be referred to as MTSM650, MTSM-700, etc.

\section{Synthesis of silica microspheres}

Silica microspheres were synthesized using the same procedure described in "General procedure" section, without the use of the titanium reagent, hence, $4.0 \mathrm{ml}$ of $4 \mathrm{~N} \mathrm{HNO}_{3}$ was mixed with a solution of $6.6 \mathrm{ml}$ tetraethyl orthosilicate (TEOS, 98\%) and $2.0 \mathrm{ml}$ ethanol and stirred vigorously for $30 \mathrm{~min}$. The microspheres were made mesoporous by surfactant templating, followed by drying, as stated in "General procedure" section. The silica samples thus formed were calcined for $6 \mathrm{~h}$ at various temperatures, namely $650,750,850$ and $950{ }^{\circ} \mathrm{C}$. Hereafter, these will be referred to as S-650, S-750, etc.

\section{Characterization}

HRTEM images were taken with a JEOL 2100 microscope operating at $200 \mathrm{keV}$. The samples were dispersed in methanol and then dried on a Holey carbon film $\mathrm{Cu}$ grid, for TEM measurements. Powder X-ray diffraction (PXRD) patterns were obtained with a Stoe STADI-P diffractometer equipped with a Mythen $1 \mathrm{~K}$ detector and using $\mathrm{Cu} \mathrm{Ka}_{1}$ radiation
(40 kV and $30 \mathrm{~mA}$ ). Samples were ground and filled into $0.7 \mathrm{~mm}$ X-ray glass capillaries. Nitrogen adsorption/desorption isotherms were conducted on a Quantachrome Autosorb $\mathrm{iQ}_{2}$, using the NLDFT method to evaluate surface area, pore volume and pore size distributions, from the adsorption branch of the isotherms [37]. XPS spectra were recorded on a Thermo Scientific spectrometer with $\mathrm{Cu} \mathrm{K} \alpha$ radiation. The analyzer was set at a pass energy of $20 \mathrm{eV}$ for high-resolution spectra of all the individual elements in each sample tested. Approximately 2-5 mg of each powder sample was mounted on a stainless-steel sample holder. The background was determined using the Shirley-type background correction, and the curves were fitted with Gaussian and Lorentzian product functions.

\section{Catalytic experiments}

The MTSM and silica samples were catalytically characterized via epoxidation of cyclohexene with TBHP according to a previously reported procedure [36, 38]. $25 \mathrm{mmol}$ of cyclohexene was mixed with $20 \mathrm{~mL}$ of decane as solvent in a batch reactor, followed by $100 \mathrm{mg}$ of MTSM catalyst. Next, approximately $7 \mathrm{~g}$ of $4 \AA$ molecular sieves were added to the reactor in order to remove moisture from the mixture. The mixture was stirred at $60{ }^{\circ} \mathrm{C}$ for $30 \mathrm{~min}$. The $5.5 \mathrm{M}$ TBHP in decane solution was dried by adding $1 \mathrm{~g}$ of molecular sieves to $5 \mathrm{ml}$ of the TBHP solution and storing overnight, prior to the reaction, to remove any adsorbed water. The reaction was initiated by adding $5.5 \mathrm{mmol}$ of the dried TBHP solution. Samples from the reaction mixture were withdrawn at relevant time periods and analyzed by gas chromatography using a GC-2014 Shimadzu Gas Chromatograph, employing a ZB-WAXplus Zebron capillary GC column. Calibration curves for TBHP, cyclohexene and cyclohexene oxide, were obtained using standard solutions, and used to determine the relevant concentrations of the reaction mixtures, via GC analysis.

\section{Results and discussion}

Formation of cristobalite phases within amorphous titanosilicate catalysts

In a previous publication, we reported how the Doehlert matrix statistical approach was used to 
optimize the synthesis conditions of mesoporous titanosilicate microspheres (MTSM), leading to the development of improved mesoporous catalysts, in a time-effective manner [36]. During this study, two synthetic parameters, i.e., surfactant weight and homogenizing temperature were changed simultaneously, in order to investigate synthesis conditions that would lead to the optimal catalyst (ESI, Table S1). Three MTSM samples were identified as superior catalysts (MTSM-750 samples 7, 3 and 2, in decreasing order of catalytic activity), based on product yield and selectivity obtained during the reaction. These samples were all calcined at $750{ }^{\circ} \mathrm{C}$, in order to remove the surfactant and oil mixture added during the synthesis for the purpose of templating. PXRD measurements performed on these titanosilicate samples revealed that the materials were completely amorphous with no indication of crystalline peaks (Fig. 1a). However, TEM imaging surprisingly indicated that the three most catalytically active samples mentioned above contained small regions of crystalline phases within the predominant amorphous phase (Fig. $1 \mathrm{~b}-\mathrm{d}$ ). One sample in particular, MTSM-750-3, showed more crystalline regions compared to any other sample, yet showed no indication of crystallinity in the PXRD within the limits of detection.

The enhanced catalytic activity of the above-mentioned MTSM samples was found to be a result of a combination of factors, such as accessible mesoporosity, high surface area and large pore diameters, together with increased amounts of isolated $\mathrm{Ti}^{4+}$ active centers. Samples with high amounts of micropores that can act as bottlenecks to prevent reagent molecules from reaching the $\mathrm{Ti}^{4+}$ active sites were found to be less catalytically active [36]. For the current study, experiments were conducted to, first, identify the crystalline phases formed during synthesis of titanosilicates and, secondly, investigate whether these had any impact on their catalytic activity.

Since sample MTSM-750-3 displayed the most prominent presence of crystals via TEM, it was chosen as a base sample for further experiments and analysis. In order to identify the crystalline component, it was necessary to increase their amount so that characteristic PXRD peaks could be observed and used for phase identification. In this regard, MTSM750-3 was synthesized several times and calcined at various temperatures from 650 to $950{ }^{\circ} \mathrm{C}$, with $50{ }^{\circ} \mathrm{C}$ increments (Fig. 2a). Since the previous catalytic experiments were conducted from samples calcined at $750{ }^{\circ} \mathrm{C}$, temperatures higher than that, i.e., 800, 850, 900 and $950{ }^{\circ} \mathrm{C}$, were used to induce formation of more crystalline materials. Lower temperatures of 650 and $700{ }^{\circ} \mathrm{C}$ were also used to determine the threshold of crystallite formation. At these lower temperatures and at $750{ }^{\circ} \mathrm{C}$, only a broad peak, characteristic of the amorphous titanosilicate was observed, similar to previous observations. A temperature of $800{ }^{\circ} \mathrm{C}$ was necessary to form a sufficient amount of crystals that can be identified via PXRD. A clear increase in crystal formation was observed with increasing calcination temperatures. At $950{ }^{\circ} \mathrm{C}$, the titanosilicate appeared to be completely crystallized with no indication of the amorphous peak (Fig. 2b).

Three different crystalline phases were identified via PXRD, as cristobalite, rutile and anatase. Among these, the predominant phase was clearly observed to be cristobalite, which is a crystalline form of silica. The other two phases; rutile and anatase, which are crystalline forms of titania, appear to be present in only small quantities. This observation reveals a couple of surprising factors with respect to phase transition in titanosilicates: First, formation of cristobalite at a temperature as low as $800{ }^{\circ} \mathrm{C}$ is highly unusual, without the addition of alkali metal salts, and secondly, cristobalite is the predominant form of crystalline material formed, even though rutile and anatase can readily form at much lower temperatures. This led to the hypothesis that some other factor could be inducing the formation of cristobalite, which was previously unknown.

In order to verify whether this effect is induced by the presence of $\mathrm{Ti}^{4+}$, silica samples were synthesized according to procedures similar to that of the titanosilicates, with the exception of the addition of Ti(IV) n-butoxide. This was to ensure that all other factors related to the synthesis were kept identical, except for the presence of $\mathrm{Ti}^{4+}$ in the silica framework. The silica samples thus formed were calcined at 750, 850 and $950{ }^{\circ} \mathrm{C}$. Unlike in the titanosilicate samples, there was no indication of crystal formation in silica until $950{ }^{\circ} \mathrm{C}$ (Fig. 2c). The predominant crystalline phase formed here was once again identified as cristobalite. Hence, in formation of cristobalite, a $150{ }^{\circ} \mathrm{C}$ difference exists between the titanosilicate and silica samples, indicating that the presence of $\mathrm{Ti}^{4+}$ may have an implication in its formation.

As stated previously, cristobalite is usually formed at $1470-1705^{\circ} \mathrm{C}$, in pure silica, and its occurrence at 

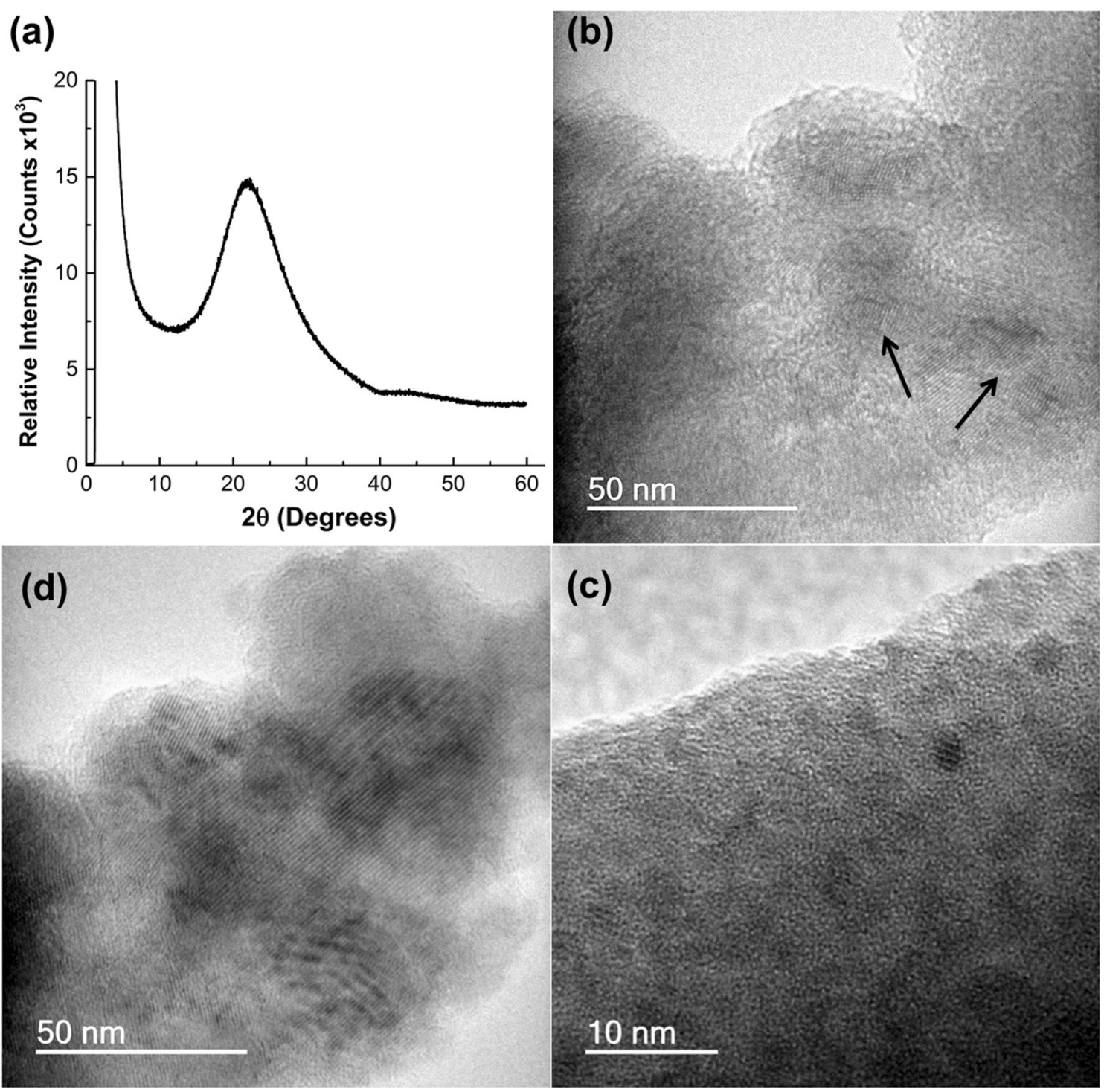

Figure 1 a PXRD data of MTSM-750-3, showing one broad peak, indicating that the material is amorphous, b, $\mathbf{c}$ and $\mathbf{d}$ TEM images of MTSM-750-7, -3, and -2 , respectively, showing the presence of crystalline phases, among the predominant amorphous phase.

$950{ }^{\circ} \mathrm{C}$ is rare, especially without the addition of alkali metals. However, it has been reported that presence of quartz crystals can significantly lower the phase transition temperature of cristobalite formation [39]. It is possible that small amounts of quartz crystals were formed during the synthesis of the silica sample, and were undetected by XRD, which in turn induced the formation of cristobalite at $950{ }^{\circ} \mathrm{C}$. Furthermore, cristobalite formation has been reported in silica sand, containing the quartz phase, calcined between 800 and $1000{ }^{\circ} \mathrm{C}$ [40], further indicating the impact of such heterogeneities on lowering phase transition temperatures, in silica.
TEM imaging conducted on the samples revealed the presence of crystals in all titanosilicate samples, except for MTSM-650 (Fig. 3). This is indicative that temperatures of at least $700{ }^{\circ} \mathrm{C}$ are necessary for the formation of cristobalite, and that the crystals formed below $800{ }^{\circ} \mathrm{C}$ are present only in trace quantities and, therefore, cannot be detected via PXRD.

\section{The effect of cristobalite formation on titanosilicate catalysis}

The fact that formation of cristobalite was confirmed via PXRD in titanosilicates at $800{ }^{\circ} \mathrm{C}$, but only at $950{ }^{\circ} \mathrm{C}$ in the corresponding silica samples, is 

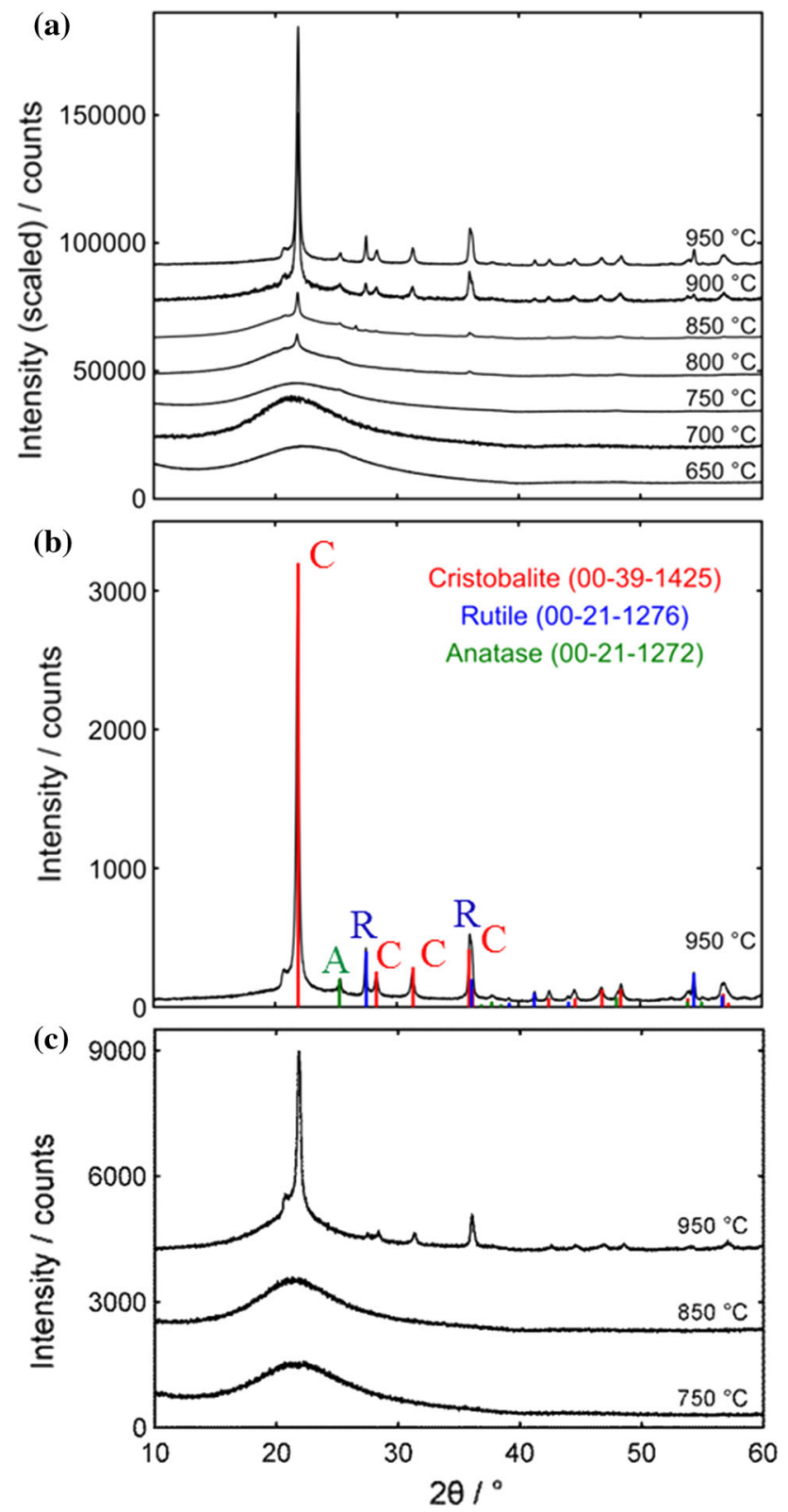

Figure 2 PXRD data for titanosilicate and silica samples calcined at different temperatures. a Propagation of crystal growth in titanosilicates MTSM-650 to -950 . b Titanosilicate MTSM-950. Sample appears completely crystallized with crystalline phases of cristobalite (C), rutile (R) and anatase (A). The predominant crystalline phase is cristobalite. c Propagation of crystal growth in silica samples S-750, S-850 and S-950.

indicative that the phenomenon is induced by $\mathrm{Ti}^{4+}$. This is surprising, as the factors that normally facilitate the formation of cristobalite at lower temperatures, such as alkali metals, were not present during the synthesis. It is known that such secondary species, such as $\mathrm{Na}^{+}$, need to be present not just as impurities, but as components in significant amounts, in order for cristobalite formation to occur around $900{ }^{\circ} \mathrm{C}$ [19]. XPS studies confirmed that there was no presence of $\mathrm{Na}^{+}$or other alkali metals in our materials and the only species present were $\mathrm{O}, \mathrm{Si}$ and $\mathrm{Ti}$, within $\mathrm{Ti}-\mathrm{O}-\mathrm{Ti}, \mathrm{Si}-\mathrm{O}-\mathrm{Si}$ and $\mathrm{Ti}-\mathrm{O}-\mathrm{Si}$ environments (ESI, Table S3, Figure S6).

The next important question is whether the cristobalite formed had any impact on the catalytic activity of titanosilicates. In order to verify this, we conducted several catalytic experiments, where cyclohexene was oxidized using TBHP in decane, and titanosilicates MTSM-650, MTSM-700, MTSM-750, MTSM-800, MTSM-850 or MTSM- $900{ }^{\circ} \mathrm{C}$ served as catalyst (Fig. 4). An equivalent silica sample S-750 was also used for comparison. Samples were taken out immediately after mixing in TBHP (which triggers the reaction), and designated as $0 \mathrm{~h}$, followed by sampling at 6 and $24 \mathrm{~h}$. The progress of the reaction was measured by the formation of cyclohexene oxide (i.e., epoxide of cyclohexene, which is the major product), with respect to TBHP (i.e., limiting reagent).

No significant product formation was observed immediately after mixing, at $0 \mathrm{~h}$. After $6 \mathrm{~h}$ of reaction a trend became evident, where the highest epoxide yield was obtained by the catalyst MTSM-700, followed by similar yields within experimental error from the MTSM-750, -650 and -800 samples. Catalysts calcined at $850^{\circ} \mathrm{C}$ and beyond had much lower epoxide yields. These observations can be explained by analyzing the nitrogen physisorption and XPS data of the samples. The BET surface area and the total pore volume decreased rapidly with increasing calcination temperature (Fig. 5a and Table 1). However, the amount of catalytically active tetrahedral $\mathrm{Ti}^{4+}$ sites, represented by the $\mathrm{Ti} 2 \mathrm{p}_{1 / 2} \%$ from XPS analysis, did not follow this trend. The sample calcined at $700{ }^{\circ} \mathrm{C}$, which had the second highest BET surface area, pore size and active sites \%, was the best catalyst at $6 \mathrm{~h}$, with $46 \%$ yield, surpassing the $650{ }^{\circ} \mathrm{C}$ sample ( $30 \%$ yield), which had the highest surface area and pore volume, and the $750{ }^{\circ} \mathrm{C}$ sample $(34 \%$ yield), which had the highest \% of active sites.

Despite having high surface area and pore volume, the MTSM-650 sample had a high percentage of micropores, which can act as bottlenecks that prevent the reagents from reaching the catalytic active sites. This leads to a reduction in overall catalytic activity and can be detected as a gating effect, which creates a 

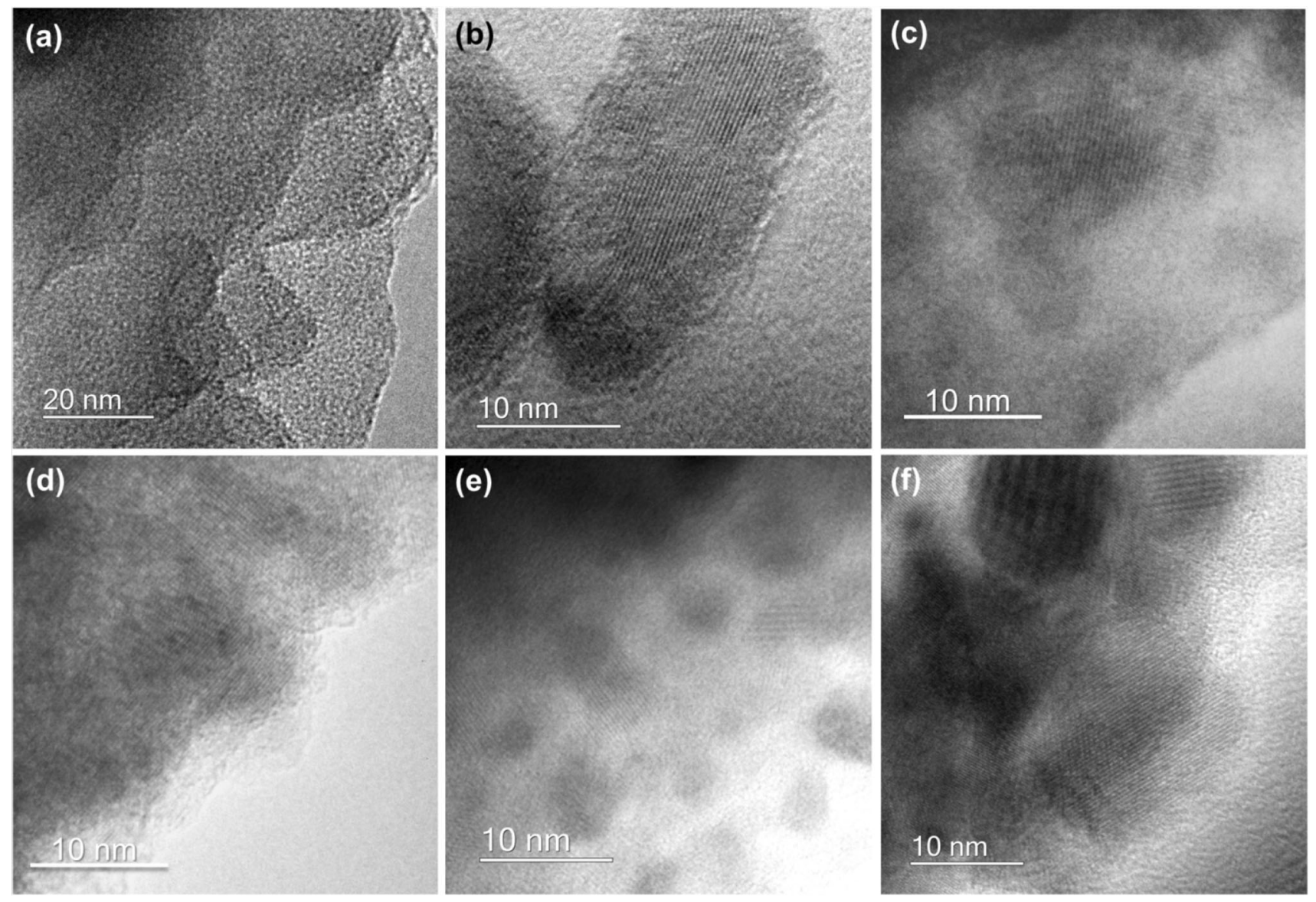

Figure 3 TEM images of titanosilicate MTSM-3 calcined at different temperatures showing formation of cristobalite. a MTSM-650, b MTSM-700, c MTSM-800, d MTSM-850,

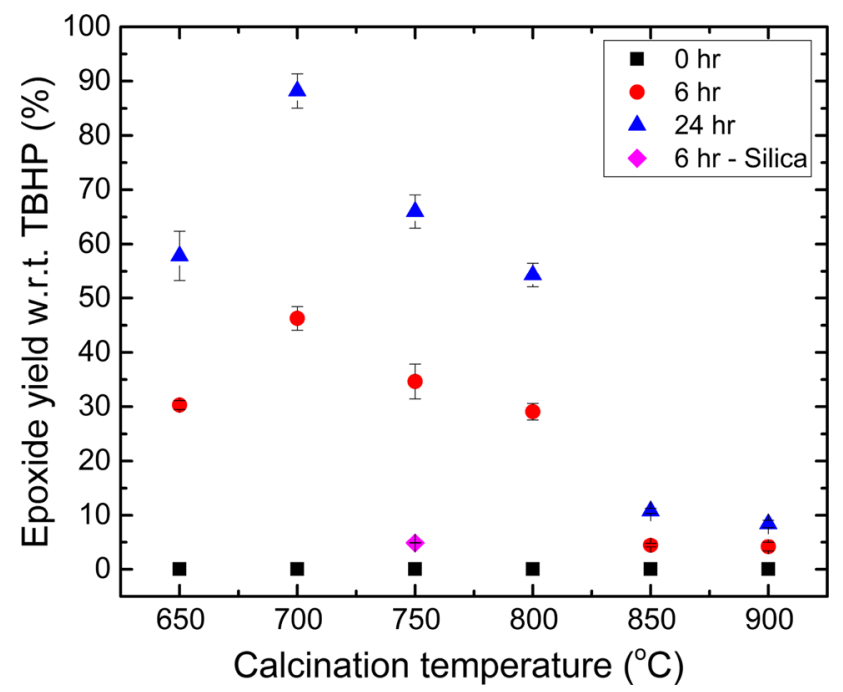

Figure 4 Catalytic results for titanosilicate samples MTSM-650 to $-900{ }^{\circ} \mathrm{C}$ and silica sample S-750, in terms of epoxide yield with respect to TBHP. e MTSM-900 and $\mathbf{f}$ MTSM-950 ${ }^{\circ} \mathrm{C}$. With the exception of (a), all other samples appear to show the presence of cristobalite.

gap between the adsorption-desorption BET isotherm branches, beyond the hysteresis loop (Fig. 5a). MTSM-650 also had less than half of the \% of active sites of MTSM-750, further explaining its relatively low activity. On the other hand, MTSM-750, despite having a high \% of active sites, had a much lower surface area and pore volume, and the combined effect was reflected in its lower catalytic activity, compared to MTSM-700. Thus, the sample with a combination of the most favorable features for catalysis appears to be the best catalyst, in line with our previous study [36]. Continuing this trend, MTSM800 gave a low yield of $29 \%$, and samples with higher calcination temperatures were shown to be even poorer catalysts. The pore network appeared to have collapsed in samples calcined at higher temperatures of 850,900 and $950{ }^{\circ} \mathrm{C}$, hindering the reagents from reaching the catalytic sites and, thus, leading to low catalytic activity. 
(a)

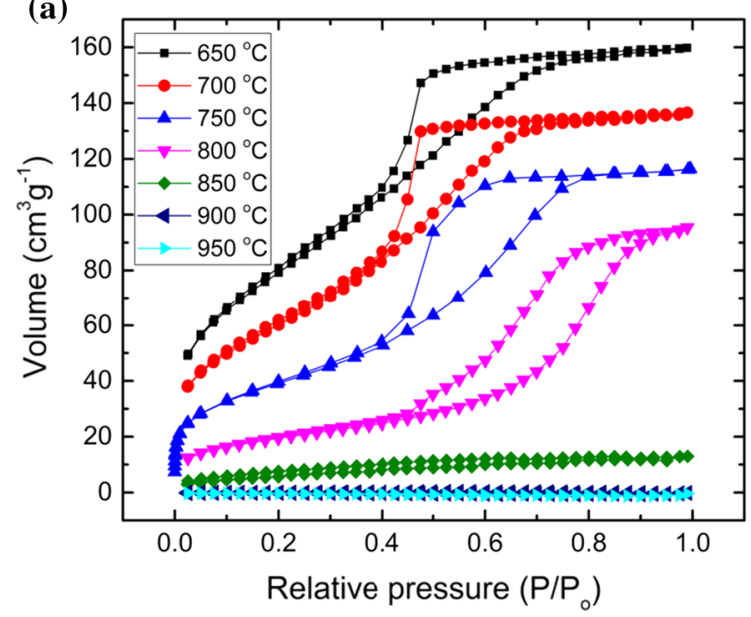

(b)

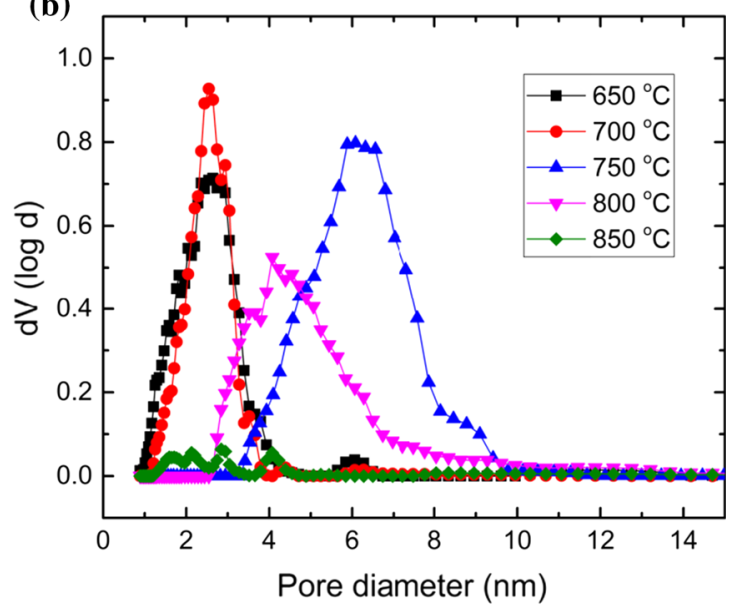

Figure 5 a Adsorption-desorption isotherms for titanosilicate samples MTSM-650, -700, -750, -800, -850, -900 and -950, analyzed using $\mathrm{N}_{2}$ at $77 \mathrm{~K}$. b Pore size distributions for the above samples calculated using the NLDFT method.

Table 1 Nitrogen physisorption and XPS data for MTSM-750-3 calcined at different temperatures

\begin{tabular}{|c|c|c|c|c|c|}
\hline $\begin{array}{l}\text { Calcination temperature } \\
\text { of MTSM samples } /{ }^{\circ} \mathrm{C}\end{array}$ & $\begin{array}{l}\text { BET surface } \\
\text { area } / \mathrm{m}^{2} \mathrm{~g}^{-1}\end{array}$ & $\begin{array}{l}\text { Total pore } \\
\text { volume } / \mathrm{ccg}^{-1}\end{array}$ & $\begin{array}{l}\text { Micropore } \\
\text { volume/\% }\end{array}$ & $\begin{array}{l}\text { Average pore } \\
\text { diameter/nm }\end{array}$ & $\begin{array}{l}\text { Tetrahedral } \\
\text { Ti } 2 p_{1 / 2} / \%\end{array}$ \\
\hline 650 & 295 & 0.239 & 37 & 2.5 & 0.65 \\
\hline 700 & 222 & 0.203 & 24 & 2.6 & 1.56 \\
\hline 750 & 142 & 0.165 & 6 & 6 & 1.7 \\
\hline 800 & 71 & 0.143 & 0 & 4.5 & 0.53 \\
\hline 850 & - & - & - & - & 0.51 \\
\hline 900 & - & - & - & - & 0.66 \\
\hline 950 & - & - & - & - & 0.78 \\
\hline
\end{tabular}

We have previously studied the kinetics of this reaction and determined that $\sim 24 \mathrm{~h}$ is sufficient for its completion under the above-mentioned reaction conditions, and hence, samples were also collected at this time. The trend in epoxide formation appeared to be similar after $24 \mathrm{~h}$, as the sample calcined at $700{ }^{\circ} \mathrm{C}$ gave the best epoxide yield of $88 \%$. This was followed by 750,650 and $800{ }^{\circ} \mathrm{C}$ samples with yields of 66,58 and $54 \%$. It is noteworthy that the latter three yields are within each other's experimental error $(\sim 17 \%)$ and are hence effectively the same. MTSM-850 and MTSM-900 samples gave rather low yields of $11 \%$ and $8 \%$, respectively, in keeping with the previous trend. The activities of each sample once again can be linked with the pore structure and the \% of active site, along with the amounts of cristobalite formed at each temperature.

It must be noted that an increasing amount of cristobalite is formed with increasing calcination temperature, according to the PXRD analysis. Even in low-temperature samples such as MTSM-650, -700 and -750 , it is reasonable to assume that cristobalite is present in smaller quantities, as evidenced by TEM, despite the absence of characteristic peaks in PXRD. As evidenced by the BET data, formation of increased amounts of cristobalite crystals causes the pore network of the materials to collapse, resulting in progressively lower catalytic activity with increasing temperature. This was particularly evident for samples calcined at $850{ }^{\circ} \mathrm{C}$ and above. Therefore, MTSM900 and $-950{ }^{\circ} \mathrm{C}$ gave very low product yields, despite having similar \% of active sites to MTSM-650. Hence, it can be concluded that an increased amount of cristobalite is detrimental to titanosilicate catalysis, as its formation leads to breakdown of the pore network. When present in smaller quantities, this effect is far less evident, and the catalytic activity is then dependent on established factors such as high surface 
area, pore volume and \% of active sites of the titanosilicate. Thus, cristobalite formation appears to have a negative effect on the catalytic activity of titanosilicates.

\section{Significance of cristobalite formation in titanosilicates at lower temperatures}

This study revealed three significant factors that are imperative to titanosilicate catalysis, particularly when the material synthesis requires a calcination step: First, formation of non-active crystalline phases, such as cristobalite, can occur at much lower temperatures than previously reported, without the addition of any alkali metals; second, $\mathrm{Ti}^{4+}$ can induce the formation of cristobalite; and third, the formation of significant amounts of cristobalite is detrimental to the catalytic activity of titanosilicates. These findings provide insight into how the process of calcination can have a significant impact on the quality control of the final product. Calcination is a step that is often overlooked during amorphous zeolite and titanosilicate production, as its purpose is to simply remove the hydrocarbon templating agents $[17,36]$. In contrast, for crystalline zeolites and titanosilicates, calcination is a means to achieve the desired framework structure and may impact catalytic activity [41, 42]. Thus, for amorphous materials, it is important to select calcination temperatures that effectively remove the templating reagents, while not causing undesirable phase transformations. As demonstrated by this study, such processes can occur in titanosilicates, even under previously unknown conditions. When considering large-scale synthesis, the presence of undesirable phases, such as cristobalite, may contribute to significant losses in catalytic activity and cause loss of efficiency in the entire manufacturing process. Therefore, careful monitoring of calcination conditions and investigating the factors that may induce such anomalies are critical in developing efficient catalysts.

\section{Conclusions}

We have demonstrated that cristobalite, a crystalline form of silica, can form in titanosilicates at relatively lower temperatures, without the presence of alkali metals, which are previously unknown conditions. PXRD studies revealed the presence of cristobalite crystals dispersed in an amorphous form of titanosilicate, MTSM, at $800{ }^{\circ} \mathrm{C}$. However, HRTEM imaging revealed that these crystals can form in smaller quantities at temperatures as low as $700{ }^{\circ} \mathrm{C}$. MTSM samples were synthesized according to previously known conditions and calcined at temperatures between 650 and $950{ }^{\circ} \mathrm{C}$, in order to investigate the propagation of cristobalite formation. With annealing temperature, increased amounts of cristobalite were formed and the materials appeared to be completely crystallized at temperatures $900{ }^{\circ} \mathrm{C}$ and above. These titanosilicates were used to catalyze the epoxidation of cyclohexene with TBHP in order to investigate the effect of cristobalite formation on catalysis. The sample calcined at $700{ }^{\circ} \mathrm{C}$ (MTSM-700) was the most effective catalyst with $88 \%$ epoxide yield with respect to TBHP, after $24 \mathrm{~h}$ of reaction conducted at $60{ }^{\circ} \mathrm{C}$, followed by MTSM-750, -650 and -800 samples. Materials calcined at higher temperatures were found to be extremely poor catalysts. The catalytic activity of these samples was influenced by their high surface area and mesopore volume, which favored the efficient transfer of reagents to the catalytically active sites. Increasing calcination temperatures allowed higher amounts of cristobalite crystals to be formed, which led to the collapse of the pore network and subsequent low catalytic activity. Investigation of such factors that lead to phase transitions in titanosilicates, under previously unknown conditions, will allow greater quality control in catalyst synthesis, via controlling calcination conditions.

\section{Acknowledgements}

The authors gratefully acknowledge the valuable contributions from Dr Steven Firth of Department of Chemistry, UCL, for assistance with TEM imaging and Mr Jim Davy of Department of Earth Sciences, UCL, for SEM imaging. The EPSRC is gratefully acknowledged for support via a "Frontier Engineering" Award, EP/K038656/1 and for funding the X-ray diffractometers, Grant EP/K03930X/1.

\section{Compliance with ethical standards}

Conflict of interest The authors declare that they have no conflict of interest. 
Electronic supplementary material: The online version of this article (https://doi.org/10.1007/s108 53-018-2869-0) contains supplementary material, which is available to authorized users.

Open Access This article is distributed under the terms of the Creative Commons Attribution 4.0 International License (http://creativecommons.org/ licenses/by/4.0/), which permits unrestricted use, distribution, and reproduction in any medium, provided you give appropriate credit to the original author(s) and the source, provide a link to the Creative Commons license, and indicate if changes were made.

\section{References}

[1] Perera AS, Coppens M-O (2016) Titano-silicates: highlights on development, evolution and application in oxidative catalysis. In: Spivey JJ (ed) Catalysis, vol 28. The Royal Society of Chemistry, pp 119-143

[2] Rocha J, Anderson Michael W (2000) Microporous titanosilicates and other novel mixed octahedral-tetrahedral framework oxides. Eur J Inorg Chem 2000:801-818

[3] Přech J (2018) Catalytic performance of advanced titanosilicate selective oxidation catalysts - a review. Catal Rev 60:71-131

[4] Gates BC, Knüpffer H (2004) Advances in catalysis, vol 48. Elsevier, New York

[5] Notari B (1993) Titanium silicalites. Catal Today 18:163-172

[6] Clerici MG (2009) Titanium silicalite-1. In: Jackson SD, Hargreaves JSJ (eds) Metal oxide catalysis. Wiley, Weinheim, pp 705-754

[7] Xiao F-S, Han Y, Yu Y, Meng X, Yang M, Wu S (2002) Hydrothermally stable ordered mesoporous titanosilicates with highly active catalytic sites. J Am Chem Soc 124:888-889

[8] van der Waal JC, Rigutto MS, van Bekkum H (1998) Zeolite titanium beta as a selective catalyst in the epoxidation of bulky alkenes. App Catal A General 167:331-342

[9] Camblor MA, Corma A, Esteve P, Martínez A, Valencia S (1997) Epoxidation of unsaturated fatty esters over largeporeTi-containing molecular sieves as catalysts: important role of the hydrophobic-hydrophilic properties of the molecular sieve. Chem Commun 0:795-796

[10] Wu P, Xu H, Xu L, Liu Y, He M (2013) Springer briefs in green chemistry for sustainability. Springer, Berlin
[11] Xiao FS, Meng X (2015) Zeolites in sustainable chemistry: synthesis, characterization and catalytic applications. Springer, Berlin

[12] Sinha AK, Seelan S, Okumura M, Akita T, Tsubota S, Haruta M (2005) Three-dimensional mesoporous titanosilicates prepared by modified sol-gel method: ideal gold catalyst supports for enhanced propene epoxidation. J Phys Chem B 109:3956-3965

[13] Kostov-Kytin V, Mihailova B, Kalvachev Y, Tarassov M (2005) Atomic arrangements in amorphous sodium titanosilicate precursor powders. Microporous Mesoporous Mater 86:223-230

[14] Naderi M, Anderson MW (1996) Phase transformation of microporous titanosilicate ETS-4 into narsarsukite. Zeolites $17: 437-443$

[15] Wang J, Zhao Y, Yokoi T, Kondo JN, Tatsumi T (2014) High-performance titanosilicate catalyst obtained through combination of liquid-phase and solid-phase transformation mechanisms. ChemCatChem 6:2719-2726

[16] Liu X, Thomas JK (1996) Synthesis of microporous titanosilicates ETS-10 and ETS-4 using solid $\mathrm{TiO}_{2}$ as the source of titanium. Chem Commun 0:1435-1436

[17] Li W, Coppens M-O (2005) Synthesis and characterization of stable hollow Ti-silica microspheres with a mesoporous shell. Chem Mater 17:2241-2246

[18] Ilyushin GD (2003) Phase relations in the $\mathrm{KOH}-\mathrm{TiO}_{2}-\mathrm{SiO}_{2}-$ $\mathrm{H}_{2} \mathrm{O}$ system at $500{ }^{\circ} \mathrm{C}$ and $0.1 \mathrm{GPa}$. Inorg Mater 39:1067-1073

[19] Cole S (1935) The conversion of quartz into cristobalite below $1000{ }^{\circ} \mathrm{C}$, and some properties of the cristobalite formed. J Am Ceram Soc 18:149-154

[20] Deo G, Turek AM, Wachs IE, Huybrechts DRC, Jacobs PA (1993) Characterization of titania silicalites. Zeolites 13:365-373

[21] Notari B (1993) Titanium silicalites. Catal Today 18:163-172

[22] Maschmeyer T (1998) Derivatised mesoporous solids. Curr Opin Solid State Mater Sci 3:71-78

[23] Notari B (1996) Microporous crystalline titanium silicates. Adv Catal 41:253-334

[24] Thomas JM (1990) The Bakerian lecture, 1990: new microcrystalline catalysts. Philos Trans R Soc Lond Ser A Phys Eng Sci 333:173-207

[25] Huybrechts DRC, Buskens PL, Jacobs PA (1992) Physicochemical and catalytic properties of titanium silicalites. J Mol Catal 71:129-147

[26] Baxter PJ, Bonadonna C, Dupree R et al (1999) Cristobalite in volcanic ash of the Soufriere Hills volcano, Montserrat, British West Indies. Science 283:1142-1145 
[27] Cristobalite (1993-2018) mindat.org http://www.mindat.org/ min-1155.html. Accessed Jan 12, 2017

[28] Horwell CJ, Williamson BJ, Llewellin EW, Damby DE, Le Blond JS (2013) The nature and formation of cristobalite at the Soufrière Hills volcano, Montserrat: implications for the petrology and stability of silicic lava domes. Bull Volcanol 75:696

[29] Lakshtanov DL, Sinogeikin SV, Bass JD (2007) High-temperature phase transitions and elasticity of silica polymorphs. Phys Chem Miner 34:11-22

[30] Roy DM, Roy R (1964) Tridymite-cristobalite relations and stable solid solutions. Am Mineral 49:952-962

[31] Evans DL (1970) Solid solution of $\mathrm{TiO}_{2}$ in $\mathrm{SiO}_{2}$. J Am Ceram Soc 53:418-418

[32] Wahl FM, Grim RE, Graff RB (1961) Phase transformations in silica as examined by continuous X-ray diffraction. Am Miner 46:196-208

[33] Rocha J, Carlos LD, Rainho JP, Lin Z, Ferreira P, Almeida RM (2000) Photoluminescence of new $\mathrm{Er}^{3+}$-doped titanosilicate materials. J Mater Chem 10:1371-1375

[34] Rainho JP, Carlos LD, Rocha J (2000) New phosphors based on $\mathrm{Eu}^{3+}$-doped microporous titanosilicates. J Lumin 87-89:1083-1086

[35] Rainho JP, Lin Z, Rocha J, Carlos LD (2003) Synthesis and luminescence of $\mathrm{Eu}^{3+}$-doped narsarsukite prepared by the sol-gel process. J Sol Gel Sci Technol 26:1005-1009
[36] Perera AS, Trogadas P, Nigra MM, Yu H, Coppens M-O (2018) Optimization of mesoporous titanosilicate catalysts for cyclohexene epoxidation via statistically guided synthesis. J Mater Sci 53:7279-7293. https://doi.org/10.1007/s10 853-018-2057-2

[37] Thommes M, Smarsly B, Groenewolt M, Ravikovitch PI, Neimark AV (2006) Adsorption hysteresis of nitrogen and argon in pore networks and characterization of novel microand mesoporous silicas. Langmuir 22:756-764

[38] Kosuge K, Singh PS (1999) Titanium-containing porous silica prepared by a modified sol-gel method. J Phys Chem B 103:3563-3569

[39] Breneman RC (2014) Material science and engineering. University of Michigan, Ph.D. thesis, http://hdl.handle.net/ 2027.42/107303

[40] Ratnawulan R, Fauzi A, Hayati AES (2018) Characterization of silica sand due to the influence of calcination temperature. IOP Conf Ser Mater Sci Eng 335:012008

[41] Inmanee T, Pinthong P, Jongsomjit B (2017) Effect of calcination temperatures and Mo modification on nanocrystalline $(\gamma-\chi)-\mathrm{Al}_{2} \mathrm{O}_{3}$ catalysts for catalytic ethanol dehydration. J Nanomater 2017:9

[42] Ma J, Chu J, Qiang L, Xue J (2013) Effect of different calcination temperatures on the structural and photocatalytic performance of $\mathrm{Bi}-\mathrm{TiO}_{2} / \mathrm{SBA}-15$. Int J Photoenergy 2013:10 